\title{
Le quartier épiscopal, campagne 2008, Byllis
} (Albanie)

Nicolas Beaudry, Pascale Chevalier et Skënder Muçaj

\section{OpenEdition}

1 Journals

Édition électronique

URL : https://journals.openedition.org/cem/11233

DOI : $10.4000 /$ cem. 11233

ISSN : 1954-3093

Éditeur

Centre d'études médiévales Saint-Germain d'Auxerre

Édition imprimée

Date de publication : 15 août 2009

Pagination : 73-76

ISSN : $1623-5770$

Référence électronique

Nicolas Beaudry, Pascale Chevalier et Skënder Muçaj, « Le quartier épiscopal, campagne 2008, Byllis (Albanie) », Bulletin du centre d'études médiévales d'Auxerre / BUCEMA [En ligne], 13 | 2009, mis en ligne le 18 octobre 2010, consulté le 22 septembre 2022. URL : http://journals.openedition.org/cem/11233; DOI : https://doi.org/10.4000/cem. 11233

Ce document a été généré automatiquement le 22 septembre 2022.

\section{(c) (i) (5)}

Creative Commons - Attribution - Pas d'Utilisation Commerciale - Partage dans les Mêmes Conditions 4.0 International - CC BY-NC-SA 4.0

https://creativecommons.org/licenses/by-nc-sa/4.0/ 


\title{
Le quartier épiscopal, campagne 2008, Byllis (Albanie)
}

\author{
Nicolas Beaudry, Pascale Chevalier et Skënder Muçaj
}

1 La dixième campagne sur le site de Byllis a occupé quatre semaines, du 5 mai au $1^{\mathrm{er}}$ juin 2008, avec une équipe de 12 spécialistes et 14 étudiants français, québécois, albanais et monténégrins, placée sous la direction conjointe de S. Muçaj et P.Chevalier ${ }^{1}$. Les travaux de terrain s'effectuent sous l'égide de l'École française d'Athènes et du ministère des Affaires Étrangères, qui les financent à parité, ainsi que de l'Institut archéologique de Tirana (cf. CEM 12, 2008).

2 La mission 2008 était centrée sur la fouille du quartier épiscopal (fig. 1). On a continué l'étude du matériel céramique et le traitement d'échantillons de sol prélevés pour des analyses archéo-environnementales, parallèlement à la consolidation du matériel métallique. Une carrière, ayant approvisionné le chantier de la muraille protobyzantine, a été brièvement explorée. Hors du complexe épiscopal, les travaux se sont limités à un accompagnement par P. Chevalier de l'équipe étudiant le pavement de mosaïque de la basilique E. M. Wurch-Kozelj et T. Kozelj ont retravaillé sur les stoai de l'agora hellénistique pour en faciliter la présentation au public par le Parc archéologique de Byllis. La basilique A, publiée dans le volume Byllis I, s'est implantée au V $V^{e}$ siècle sur l'extrémité ouest de l'ancienne stoa nord. L'étude du phasage de cette dernière a permis d'affiner la chronologie des constructions antérieures à l'église et les remaniements nécessités par celle-ci puis par sa phase de développement du $\mathrm{VI}^{\mathrm{e}}$ siècle. Ces éléments ont été intégrés au manuscrit Byllis I qui a été déposé fin septembre à l'EFA.

Fig. 1 - Byllis, plan schématique de la cathédrale (basilique B) et du quartier épiscopal : implantation des fouilles et nettoyages en 2008 (del. M. Wurch-Kozelj).

3 Dans son dernier état bâti, le quartier épiscopal, au sud-est de la cathédrale, se déploie autour du croisement de deux axes perpendiculaires (fig. 1). Le premier est un ancien decumanus hellénistique, qui dessert d'ouest en est les annexes liturgiques de la cathédrale et se poursuit jusqu'au sommet de la colline, débouchant sur le cardo qui 
longe le rempart du VI ${ }^{\mathrm{e}}$ siècle. Le second axe court du nord au sud en contrebas; entre les deux, une paroi de rocher détermine une partie haute qui suit la crête et domine une partie basse en fort dénivelé vers l'ouest. À la rencontre de ces axes, la cour L desservait des espaces dépendant du complexe épiscopal. À l'est, l'accès au quartier était contrôlé par un porche ou un double portail ; dans l'angle nord-est de la cour, une grande pièce barlongue empiétait largement sur le decumanus, formant un vestibule à la suite du portail. Au sud, les murs de la partie haute du quartier ont été écrêtés jusqu'à un aménagement rupestre, dont le front de taille méridional bordait un autre decumanus; deux pièces, portées par un mur de soutènement dans la cour, prolongeaient vers l'ouest cette partie haute du quartier. La cour était limitée au nord par l'entrepôt 33 et par des pièces qui prolongent le groupe cathédral vers l'est. Le grand bâtiment K-P, construit sur deux niveaux, la fermait au sud. Son rez-de-chaussée comprenait cinq pièces (K1-5) ouvertes à l'origine sur la cour : la pièce orientale K1 (écurie ?) a livré une mangeoire et K2 un moulin à olives. La façade méridionale du bâtiment comprenait deux pièces d'angle flanquant un espace barlong $\mathrm{P} 1$, où se sont succédé des foyers (cuisine ?). Au sud-est, un mur coudé formait clôture. La cour fut close à l'ouest par le bâtiment tardif D.

Fermée par une porte dans son angle nord-ouest mais pourvue d'un accès direct au cardo du rempart, la cour L permettait donc le transit des denrées stockées dans les magasins situés à proximité, l'approvisionnement en matières premières et la distribution des produits des industries viticole et oléicole, ainsi que la circulation et l'entretien des bêtes. Depuis 2004, nous cherchons à préciser le plan, la chronologie et les fonctions des espaces d'un quartier dépendant de l'épiscopat, mais coupé du domaine liturgique et consacré à l'habitat et à des activités économiques ${ }^{2}$.

Dans la continuité des campagnes précédentes, on a poursuivi l'exploration des bâtiments N (pièces N2 et N5) et K-P (pièces K4 et P1A), qui bordent la cour L au nord et au sud. Les pièces $\mathrm{N} 2$ et N5, déjà partiellement explorées en 2007 (cf. Cem 12), s'avèrent être respectivement un entrepôt (chais?) et un vaste fouloir à vin. La toiture charpentée de $\mathrm{N} 2$ reposait sur un double arc diaphragme supporté par un pilier central; une banquette est adossée au mur est et plusieurs pithoi intérieurement bituminés ont été fouillés en rangées (fig. 2). La pièce N5 comprenait, outre le bassin sud-est, deux autres bassins rectangulaires plus grands au nord, communiquant d'est en ouest et accessibles par un escalier permettant d'y jeter le raisin; quatre pithoi alignés contre la paroi orientale réunissaient les aménagements viticoles (fig. 2). 


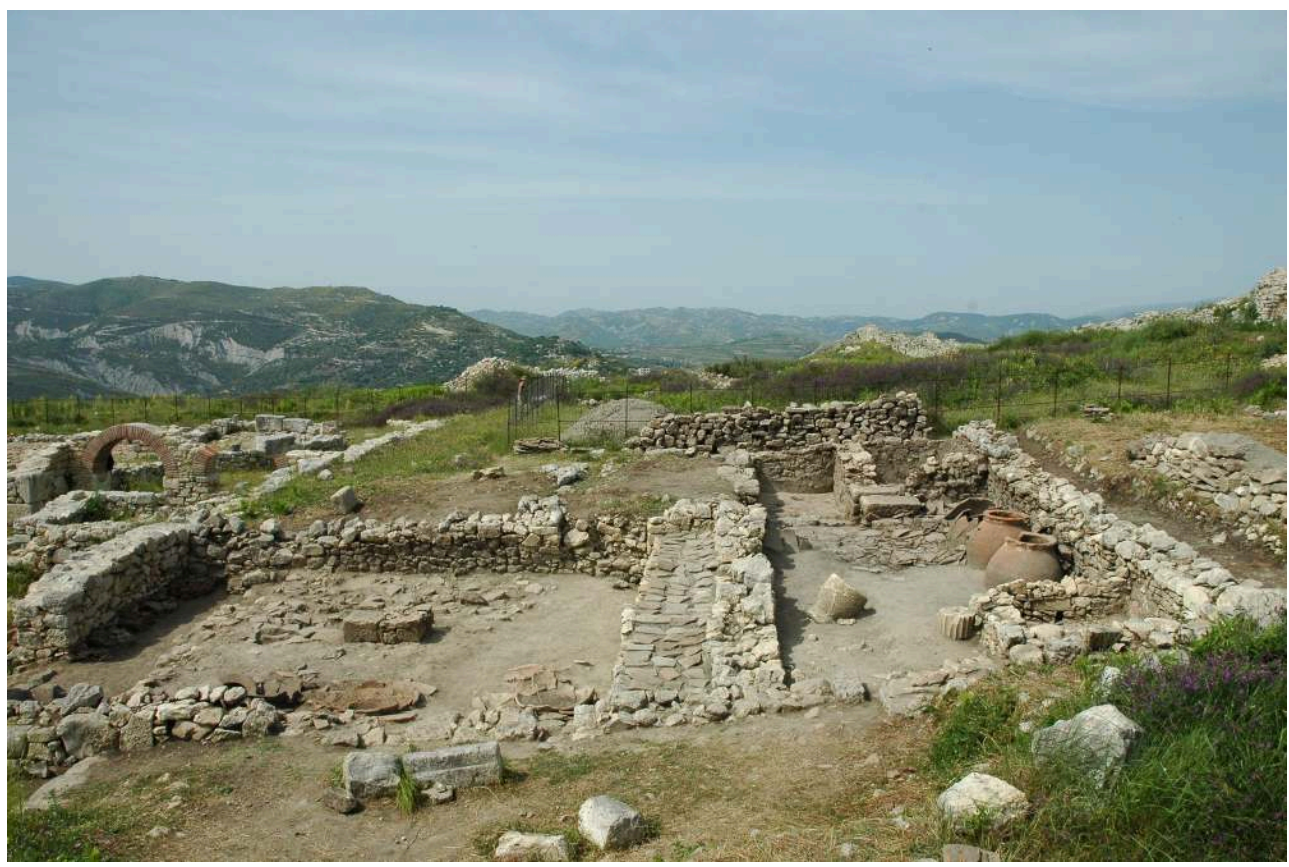

Fig. 2 - Byllis, le bâtiment N en fin de fouille, vers le nord-nord-est (cl. N. Beaudry).

La fouille de la pièce $\mathrm{K} 4 \mathrm{a}$ atteint le dernier niveau de sol sous l'effondrement de l'étage, confirmant l'existence d'une porte centrale dans le mur sud, sans équivalent connu dans les autres espaces nord du bâtiment. Précédée d'un seuil, elle est partiellement obstruée au sud par un escalier dans P1A; un étroit passage est préservé. Une porte nord, axiale (contrairement aux portes de K1-2-3), est bouchée dans le dernier état. Si les proportions de $\mathrm{K} 4$ répondent à celles de $\mathrm{K} 1$ et $\mathrm{K} 2$, son plan présente des caractères originaux et la pièce a connu d'importantes transformations.

On a repris en 2008 le nettoyage et la fouille de la partie occidentale de P1 (P1A) (fig. 1), interrompue en 2002 sur la toiture effondrée. Le mobilier calcaire y a été documenté sekôma, mortier, bassin rectangulaire. Sous les tuiles, on a retrouvé les niveaux déjà connus de sédiments noirs. Un sondage le long du mur sud a livré un canal d'évacuation des eaux usées, se poursuivant dans la porte, et un dallage de grandes pierres qui s'étend sous l'escalier tardif menant à K4.

\section{NOTES}

1. L'équipe était composée de : Skënder Muçaj (Institut archéologique de Tirana), chef de mission; Ylli Cerova, céramologue (IAT) ; Skënder Bushi et Syela Xhyeri, archéologues (IAT) ; Mariglen Meshini, Ornela Durmishaj, Berijan Premto, Klodian Velo, Brunilda Pregu, Dritan Nano, Era Xhaferaj, Besmira Shkurti, étudiants (université de Tirana) et Anton Lulgjuraj, étudiant (université de Podgorica, Monténégro); Agron 
Islami, restaurateur et Solinda Kamani, archéologue (Institut des monuments culturels de Tirana); Eduart Bitri, restaurateur du métal (Galerie nationale de Tirana); Pascale Chevalier (université BlaisePascal/Clermont-Ferrand II et UMR 5594 - ARTeHIS, CNRS), chef de mission; Nicolas Beaudry (université du Québec à Rimouski), responsable de la fouille du quartier épiscopal; Michel Bonifay, céramologue (CNRS, UMR 6573 - CCJ) ; Tony Kozelj et Manuela WurchKozelj, architectes de l'EFA ; Etleva Nallbani, étude des objets métalliques (EFR); Nadia Saint-Luc (Saint-Étienne); Sébastien Champeyrol, Geneviève Gascuel, Catherine Dupont, Julie Viriot, Chantal Gagné, étudiants (université de Clermont-Ferrand II et de Montréal).

2. Outre la notice parue dans le Bulletin du Centre d'études médiévales $\begin{array}{llll}\text { d'Auxerre, } 12 & \text { (2008), p. 63-68 [http://cem.revues.org/ }\end{array}$ document6512.html], cf. par exemple, en tout dernier lieu : N. BEAUDRY, «Le secteur sud-est du quartier épiscopal », in P. CHEVALIER et alii, «Byllis », Rapports 2005. Bulletin de correspondance hellénique, 130, 2006 (2008), p. 845-851.

INDEX

Mots-clés : quartier épiscopal, basilique

Index géographique : Albanie/Byllis 